\title{
Síndromes geriátricos, los grandes ausentes en los índices de comorbilidad usados para determinar el riesgo de desarrollar demencia
}

\section{Geriatric syndromes, noticeably absent conditions in comorbidity indexes used when determining the risk for developing dementia}

Correspondencia María del Pilar Valeria Luna Benites mapi.lb1295@gmail.com

Recibido: 25/06/2018 Aprobado: 25/07/2018

Citar como: Luna $M$, Casas A, Antón K, Bruiget S, Burneo MC Campos MI, et al. Sindromes geriátricos, los grandes ausentes en los indices de comorbilidad usados para determinar el riesgo de desarrollar demencia. Acta Med Peru. 2018;35(3):189-90

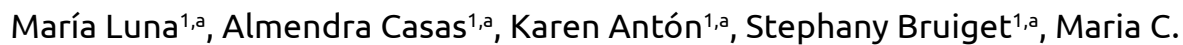
Burneo ${ }^{1, a}$, María I. Campos ${ }^{1, a}$, Fernando M. Runzer-Colmenares ${ }^{1, b}$

1 Universidad Científica del Sur. Lima, Perú

a Estudiante de Medicina Humana, b Médico geriatra

\section{Sr. Editor:}

El principio primario de la geriatría es el estudio del descenso de la capacidad de reserva homeostática de todos los sistemas orgánicos que ocurren mientras se envejece (homeoestenosis). Este declive es el resultado de la exposición a múltiples factores de riesgo individuales y de la presencia de comorbilidades crónicas. Un síndrome geriátrico (SG) es la presentación de uno o varios síntomas únicos y/o atípicos, con alta prevalencia en esta población, generados por múltiples factores y enfermedades; su relevancia está directamente relacionada con el impacto en la calidad de vida del paciente y la discapacidad es una constante amenaza a su independencia ${ }^{[1]}$.

Una comorbilidad es aquella condición médica preexistente en el paciente que puede ser identificada al momento o después del diagnóstico de una enfermedad pero que no es consecuencia de esta última. Se habla de multimorbilidad cuando hay dos o más patologías crónicas. El número de comorbilidades ha sido utilizado frecuentemente como un indicador de desenlaces negativos en geriatría; no obstante, los SG generalmente no son incluidos en dichos índices pese a que muchas veces son los determinantes esenciales en la salud de los adultos mayores ${ }^{[2]}$.

En la actualidad, los trastornos neurocognitivos (TNC) son una condición importante asociada con comorbilidad y SG, y generan desenlaces negativos como discapacidad y muerte, provocando altos costes sanitarios. Los índices de comorbilidad se asocian con una alta incidencia de demencia; empero, no se ha encontrado estudios que relacionen la suma de SG como factor de riesgo para desarrollar demencia, aun cuando muchos de ellos logran tener una relación individual con esta. El objetivo del presente estudio es presentar el avance preliminar de una investigación que trata sobre el impacto de sufrir múltiples síndromes geriátricos y el riesgo de TNC ${ }^{[3]}$.

Como avance preliminar de un estudio de cohortes realizado en el Centro Médico Naval del Perú entre los años 2010 y 2015 se hizo seguimiento a 1897 adultos mayores con el fin de determinar los factores asociados a fragilidad y TNC. Para ello, construimos una variable categórica donde presentamos las comorbilidades como una acumulación, categorizándolas en cero (0), una (1), dos (2) y tres o más comorbilidades $(\geq 3)$. Se excluyeron los datos de los participantes con diagnóstico de TNC al inicio del estudio ( $n=335$ ). Se definió TNC a un puntaje del Montreal Cognitive Assessment (MoCA) de 25 o menos durante el seguimiento, según lo descrito en la versión en español ${ }^{[4]}$.

Las comorbilidades que incluimos fueron: hipertensión, diabetes, enfermedad pulmonar obstructiva crónica, insuficiencia vascular, dislipidemia, infección urinaria, artrosis, cardiopatía isquémica, obesidad, 
Tabla 1. Análisis descriptivo y multivariado: Riesgo de aparición de trastornos neurocognitivos según número de comorbilidades y síndromes geriátricos $(\mathrm{n}=1562)$.

\begin{tabular}{|c|c|c|c|}
\hline \multirow{2}{*}{ Variable } & $\begin{array}{c}\text { Puntaje MoCA } \\
x \pm D E\end{array}$ & \multicolumn{2}{|c|}{$\begin{array}{l}\text { Regresión de Cox } \\
\text { HR (IC95\%) }\end{array}$} \\
\hline & Al inicio del seguimiento Al final del seguimiento & Modelo crudo & Modelo ajustado \\
\hline
\end{tabular}

Número de síndromes geriátricos

$\begin{array}{ccccc}0 & 26,79 \pm 2,70 & 26,49 \pm 2,12 & \text { Referencia } & \text { Referencia } \\ 1 & 27,32 \pm 1,93 & 26,17 \pm 2,10 & 1,05(0,92-1,11) & 1,01(0,91-1,10) \\ 2 & 22,98 \pm 2,70 & 18,16 \pm 1,33 & 1,09(1,02-1,15)^{*} & 1,06(1,01-1,14)^{*} \\ \geq 3 & 18,54 \pm 1,29 & 15,96 \pm 1,31 & 1,14(1,04-1,17)^{*} & 1,11(1,02-1,12)^{*}\end{array}$

Número de comorbilidades

0

1

2

$\geq 3$
$25,99 \pm 4,09$

$26,29 \pm 3,54$

$23,92 \pm 4,55$

$21,59 \pm 5,68$
$24,49 \pm 1,31$

$24,38 \pm 1,92$

$21,39 \pm 1,70$

$19,12 \pm 1,23$
Referencia

$1,04(0,93-1,13)$

$1,05(1,04-1,12)^{*}$

$1,27(1,14-1,44)^{*}$
Referencia

$0,99(0,91-1,13)$

$1,06(0,91-1,24)$

$1,06(1,01-1,12)^{*}$

* Estadísticamente significativo (valor de $p<0,05$ ).

MoCA: Montreal cognitive assessment; x: media; DE: desviación estándar; HR: hazard ratio; IC95\%: intervalo de confianza al $95 \%$.

enfermedad cerebro vascular e insuficiencia cardiaca. Además, incluimos a SG como caídas, polifarmacia, fragilidad física, incontinencia urinaria, trastorno de la marcha, dependencia funcional y depresión; categorizadas similarmente a las comorbilidades. Se utilizó a su vez la escala MoCA durante todo el seguimiento para poder evaluar el deterioro cognitivo.

En la tabla 1 vemos que la media del puntaje de MoCA al final del seguimiento es menor en los grupos con más SG y comorbilidades. En relación con ello, los promedios más bajos de MoCA estuvieron correspondieron a los participantes con dos o tres o más SG o comorbilidades. Cabe destacar que los promedios de MoCA fueron menores en los participantes con múltiples SG en comparación con aquellos con tres o más comorbilidades.

Adicionalmente, se realizó una regresión de Cox para evaluar el riesgo de TNC y encontramos que el hazard ratio tendía a incrementarse según el número de SG y comorbilidades. En el análisis ajustado encontramos que tener dos SG incrementaba el riesgo de TNC en 1,06 veces y tener tres o más SG lo hacía en 1,12 veces, comparados con los participantes sin SG, independientemente del número de comorbilidades. Además, vimos que el riesgo fue mayor en comparación a lo encontrado en el análisis de número de comorbilidades.

Conclusión que tener tres o más síndromes geriátricos aumentaba el riesgo de TNC en un $12 \%$, más que tener tres o más comorbilidades que sólo aumentaba el riesgo de TNC en un 6\%; por lo tanto, en el manejo de los problemas cognitivos, debería darse más importancia a la detección precoz de los síndromes geriátricos y el control de comorbilidades, por la estrecha relación del aumento de riesgo de TNC. Además, en pacientes adultos mayores se debe cuantificar la cantidad de síndromes geriátricos ya que el acúmulo de los mismos aumentaría el riesgo de padecer TNC, por lo que se podría considerar incluir el tamizaje de SG en la valoración cognitiva de adultos mayores ${ }^{[5]}$.

\section{REFERENCIAS BIBLIOGRÁFICAS}

1. Olde Rikkert MG, Rigaud AS, van Hoeyweghen RJ, de Graaf J. Geriatric syndromes: medical misnomer or progress in geriatrics? Neth J Med. 2003;61(3):83-7.

2. Ording AG, Sørensen HT. Concepts of comorbidities, multiple morbidities, complications, and their clinical epidemiologic analogs. Clin Epidemiol. 2013;5:199-203.

3. Roffman CE, Buchanan J, Allison GT. Charlson comorbidities index. J Physiother. 2016;62(3):171.

4. Runzer-Colmenares FM, Urrunaga-Pastor D, Aguirre LG, ReateguiRivera CM, Parodi JF, Taype-Rondan A. Frailty and vulnerability as predictors of radiotoxicity in older adults: A longitudinal study in Peru. Med Clin (Barc). 2017;149(8):325-30.

5. Ferrucci L, Orini S. Comprehensive geriatric assessment: an updated perspective. En: Pilotto A, Martin FC. Comprehensive geriatric assessment. Cham, Switzerland: Springer International Publishing; 2018. p. 1-10. 